\title{
BMJ Open Association between plasma exosome neurogranin and brain structure in patients with Alzheimer's disease: a protocol study
}

\author{
MengFei He (D) , ${ }^{1}$ Li Sun, ${ }^{1}$ Wenhui Cao, ${ }^{1}$ Changhao Yin, ${ }^{1,2}$ Wenqiang Sun, ${ }^{1}$ \\ Ping Liu, ${ }^{1}$ Lin Tan, ${ }^{1}$ Zheng Xu, ${ }^{1}$ Weina Zhao ${ }^{1,2}$
}

To cite: He M, Sun L, Cao W, et al. Association between plasma exosome neurogranin and brain structure in patients with Alzheimer's disease: a protocol study. BMJ Open 2020;10:e036990. doi:10.1136/ bmjopen-2020-036990

- Prepublication history for this paper is available online. To view these files, please visit the journal online (http://dx.doi org/10.1136/bmjopen-2020036990).

Received 20 January 2020 Revised 28 May 2020 Accepted 18 July 2020
A) Check for updates

(C) Author(s) (or their employer(s)) 2020. Re-use permitted under CC BY-NC. No commercial re-use. See rights and permissions. Published by BMJ.

${ }^{1}$ Department of Neurology, HongQi Hospital, Mudanjiang Medical University, Mudanjiang, China

${ }^{2}$ Heilongjiang Key Laboratory of Ischemic Stroke Prevention and Treatment, Mudanjiang Medical University, Mudanjiang, China

Correspondence to

Dr Weina Zhao;

weinzhao@126.com

\section{ABSTRACT}

Introduction Neurogranin is known to be significantly elevated in patients with Alzheimer's disease (AD) and may be an effective clinical predictor of cognitive decline and neurodegeneration. Amnestic mild cognitive impairment (aMCl) is an intermediate disease state between normal cognitive ageing and dementia, the latter of which can easily revert to $A D$. There remains significant uncertainty regarding the conversion of $\mathrm{aMCl}$ to $\mathrm{AD}$, and therefore, elucidating such progression is paramount to the field of cognitive neuroscience. In this protocol study, we therefore aim to investigate the changes in plasma neurogranin in the early stage of $A D$ and the mechanism thereof regarding the cognitive progression towards AD.

Methods and analysis In this study, patients with aMCI and $A D$ patients ( $\mathrm{n}=70$ each) will be recruited at the memory clinic of the Department of Neurology of Hongqi Hospital affiliated with the Mudanjiang Medical University of China. Healthy older controls $(n=70)$ will also be recruited from the community. All subjects will undergo neuroimaging and neuropsychological evaluations in addition to blood collection at the first year and the third year. We hope to identify a new biomarker of cognitive decline associated with $A D$ and characterise its behaviour throughout the progression of $\mathrm{aMCl}$ to $\mathrm{AD}$. This work will reveal novel targets for the therapeutic prevention, diagnosis and treatment of $A D$. The primary outcome measures will be (1) neuropsychological evaluation, including Mini-Mental State Examination, Montreal Cognitive Assessment, Clinical Dementia Rating scale, Shape Trail Test-A\&B, Auditory Verbal Learning TestHuaShan version; (2) microstructural alterations and hippocampal features from MRI scans; and (3) neurogranin levels in the neuronal-derived exosomes from peripheral blood samples.

Ethics and dissemination The ethics committee of the Hongqi Hospital affiliated with the Mudanjiang Medical University of China has approved this study protocol. The results will be published in peer-reviewed journals and presented at national or international scientific conferences. Trial registration number ChiCTR2000029055.

\section{INTRODUCTION}

With the ageing global population, the incidence of dementia is increasing and is posing
Strengths and limitations of this study

- A strength of this study is the use of multimodal marker types (clinical, blood and imaging markers) to predict conversion from normal to mild cognitive impairment, which will provide references for early diagnosis of Alzheimer's disease (AD).

- This study may simplify the operations associated with sample collection by examining plasma exosome neurogranin as cerebrospinal fluid is difficult to obtain.

- The relatively short follow-up duration (3 years) of this study allows longitudinal data to be analysed only in an exploratory way.

- The experiments rely on the key laboratory of prevention and treatment of ischaemic stroke in Heilongjiang Province to ensure technical feasibility.

- The study results will be limited by the research environment in that individuals will be recruited from memory clinics and cold regions, without nationwide population-based samples.

a significant burden on the worldwide social economy. For example, the number of people living with dementia in China alone will reach 28 million by $2050 .{ }^{1}$ Alzheimer's disease (AD) is the most common cause of dementia in the elderly, but unfortunately there are currently no drugs to fully treat or cure $\mathrm{AD}$ amyloid pathology. In addition to amyloid and tau proteins, synaptic loss and/or dysfunction are also important pathophysiological features in $\mathrm{AD}$ and play a key role in the cognitive decline and neurodegeneration of patients with $\mathrm{AD} .{ }^{2}$ Detecting and elucidating the mechanism of synaptic dysfunction in the pathogenesis of $\mathrm{AD}$ will have a profound impact on the early diagnosis and treatment of AD.

Neurogranin, a postsynaptic protein distributed in dendrites, is secreted mainly by excitatory neurons in the neocortex and hippocampus and participates in the regulation of synaptic plasticity such as long-term 
potentiation and long-term depression that are key to memory consolidation. ${ }^{3}$ It has been shown that neurogranin is significantly elevated in patients with $\mathrm{AD}$ and that the levels of neurogranin and tau protein are significantly correlated in patients with $\mathrm{AD} .{ }^{4}$ Furthermore, the amount of neurogranin in cerebrospinal fluid (CSF) can also differentiate early patients with $\mathrm{AD}$ from healthy controls and predict potential cognitive impairment in normal controls in addition to the more extreme impairment in patients with dementia. ${ }^{4}$ The level of neurogranin in typical patients with $\mathrm{AD}$ is higher than that in patients with atypical $\mathrm{AD}^{5}$ and gradually increases over the course of the disease (dementia>mild cognitive impairment $>$ healthy control). ${ }^{6}$ In addition, it has further been found that patients with progressive mild cognitive impairment (MCI) exhibit a higher level of CSF neurogranin than those with stable MCI. ${ }^{78}$ Along these lines, it has been suggested that a higher CSF neurogranin level is inversely correlated with baseline memory function in patients with MCI. In fact, neurogranin level is a predictor of the progressive decline of memory and executive function in patients with MCI, but not in elderly control subjects, indicating a potential disease-specific effect. $^{9}$ In support of this idea, CSF neurogranin was found to increase gradually in a normal elderly population without cognitive impairments, but did not change in patients with $\mathrm{MCI}$ or $\mathrm{AD}$, which suggests that this is an early indicator. ${ }^{8}$

It is apparent that neurogranin can predict cognitive decline and neurodegeneration; however, such exemplary studies have focused on CSF content. Nevertheless, although CSF biomarkers are highly accurate for the diagnosis of $\mathrm{AD}$ and MCI, the associated tissue collection methods are more traumatic to patients. On the other hand, using peripheral blood biomarkers for $\mathrm{AD}$ diagnosis has the advantages of being less traumatic, being easy to sample and being more generalisable across widespread populations and facilities, but with the disadvantage of exhibiting a lower sensitivity. Moreover, direct detection of plasma neurogranin content shows that there is no significant difference between patients with $\mathrm{AD}$ and healthy controls, and that there is no correlation between the neurogranin content in plasma and $\mathrm{CSF}^{10}$ One study used mass spectrometry to detect neurogranin polypeptides in plasma and CSF and found that there were unique traces in both sources, indicating that different information is stored in each fluid. Thus, identifying more sensitive biomarkers of $\mathrm{AD}$ in the peripheral blood has become a key problem that needs to be solved.

Although no change has been previously found in the level of plasma neurogranin in the above studies, with the development of neuron-derived exosome (NDE) detection technology in plasma, new insights into the pathogenesis of $\mathrm{AD}$ can now be observed. For example, we can now detect the changes of neurodegenerationrelated neuronal proteins in patients with $\mathrm{AD} .{ }^{11}{ }^{12}$ Unlike the increase of neurogranin in the CSF of patients with $\mathrm{AD}$, the neurogranin in NDEs exhibits the opposite trend which is likely due to the transport of neurogranin from plasma into the CSF. It has also been reported that amyloid protein, tau protein and cathepsin in plasma NDEs of a normal elderly population gradually increased over the course of 8 years, while neurogranin gradually decreased, but was still far from the level of patients with $\mathrm{AD} .{ }^{13}$ Furthermore, recent work found that NDEs in plasma decreased in patients with $\mathrm{AD}$ throughout the disease and were even suppressed for several years before disease onset. ${ }^{14}$ Although we can detect neurogranin in plasma, few studies have examined neurogranin NDEs in patients with $\mathrm{AD}$ and focus mainly on the dementia stage of $\mathrm{AD}$, ignoring the early disease stages and their correlation with cognitive decline and neurodegeneration. Therefore, it is necessary to longitudinally investigate the changes of neurogranin in plasma NDEs at different stages of $\mathrm{AD}$ (ie, aMCI and dementia).

Moreover, in the normal elderly population, a higher level of neurogranin has been shown to be associated with a smaller hippocampal volume. ${ }^{3}$ Neurogranin in the CSF is also associated with brain atrophy, including that of the whole brain, hippocampus, parahippocampal gyrus and entorhinal cortex in patients with AD dementia. ${ }^{4}$ Specifically, it has been reported that CSF neurogranin at the baseline stage of MCI leads to a progressive decrease in cortical glucose metabolism and hippocampal volume in patients with MCI. ${ }^{7}$ Neuroimaging technologies such as MRI are important developments in modern medicine and have been widely used in the study of various neuropsychiatric diseases. Previous studies have suggested that the degree and rate of hippocampal atrophy are early markers of incipient memory decline and dementia. ${ }^{15}$ Our team observed that this trend varies across the hippocampus subfields and further illustrates that subjective cognitive decline is the preclinical stage of $\mathrm{AD}$ earlier than aMCI.

For early diagnosis, we will combine the volume of the whole brain and hippocampal subregions with plasma neurogranin detection to analyse the correlation between plasma NDEs and the hippocampal volume in patients with $\mathrm{AD}$. The results of this work may provide scientific insights for the more effective prevention, diagnosis and treatment of $\mathrm{AD}$.

\section{METHODS}

The study design for the current prospective trial is described in figure 1.

\section{Subjects}

We will recruit patients with $\mathrm{AD}$ dementia $(\mathrm{n}=70)$ and aMCI $(n=70)$ from a memory clinic in HongQi Hospital affiliated with the Mudanjiang Medical University in China, while the control group $(n=70)$ will be recruited from the local community. After obtaining informed consent, neurologists will collect a variety of neurological assessments and neuroimaging data as described in subsequent sections within 3 days of being enrolled. 


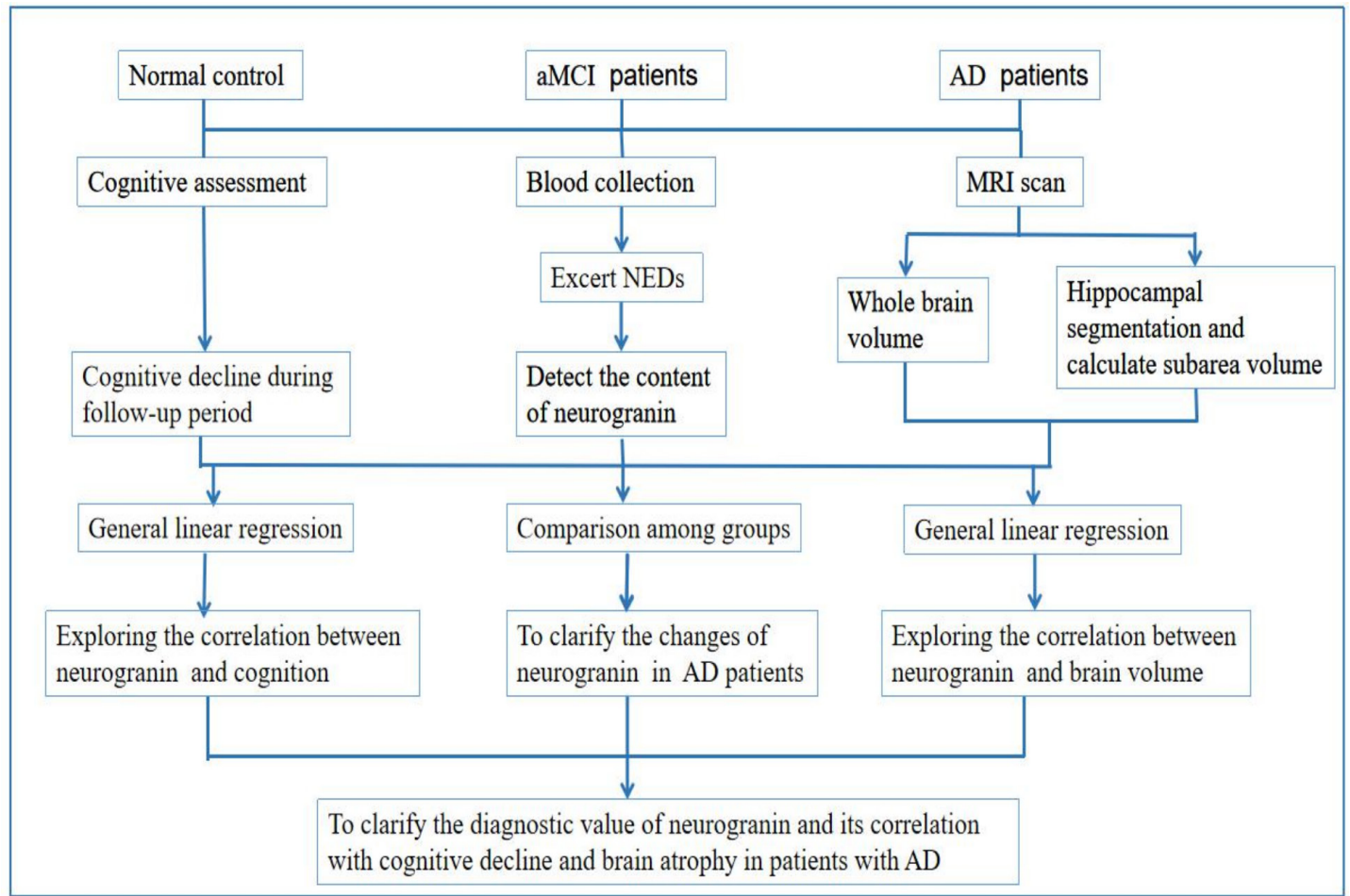

Figure 1 Flowchart of this study. AD, Alzheimer's disease; aMCl, amnestic mild cognitive impairment; NEDs, neuronal-derived exosomes.

The inclusion criteria for enrolment in this will be different for patients with aMCI and AD. The criteria for admission of patients with aMCI are based on the National Institute of Aging Alzheimer's Association 2011 diagnostic criteria and Petersen diagnostic criteria, and are as follows: (1) >65 years old, right-handed, Han nationality; (2) self-reported memory impairment; (3) a single or multidomain cognitive decline, abnormal objective memory impairment documented by memory test scores falling 1.5 SD below the age and education matched-specific norms; (4) objective evidence of memory impairment (auditory word memory test); (5) a Clinical Dementia Rating (CDR) score of 0.5; (6) no history of other diseases that cause cognitive impairment.

Patients with $\mathrm{AD}$ were enrolled according to the National Institute of Aging Alzheimer's Disease Association 2011 diagnostic criteria and are as follows: (1) self-reported memory impairment (or reported by an informed person); (2) cognitive impairment, impairment of occupational, social or daily living abilities; (3) objective evidence of memory impairment (auditory word memory test); (4) a CDR score of 1.0; (5) AD diagnosis according to the NIA-AA diagnostic criteria; (6) no history of other diseases that cause cognitive impairment.

\section{Patient and public involvement}

Participants will be consecutively recruited through standardised public advertisements and through referrals from general physicians, memory clinics and informants. Residents who meet the inclusion criteria will be recruited and written informed consent will be acquired from each subject before enrolment. Patients and the public will not be involved in the development of the research question or the design of the study. Patients will not be involved in the recruitment of participants or the conduct of the study. The general results will be disseminated to participants through public education activities.

\section{Sample size estimation}

The PASS V.15.0.5 software was used to estimate the sample size needed for our study. To obtain a statistical power (1- $\beta$ ) of $80 \%$, a was set to 0.05 and the rate of loss to follow-up was set to $20 \%$. Using information from previous CSF-focused studies and these values, we found that we will require 65 individuals per experimental group and therefore will recruit 70 per group to account for potential dropout over the course of the study.

\section{Neuropsychological assessments}

All subjects will receive a standardised clinical and neuropsychological evaluation that will include the Mini-Mental State Examination (MMSE), Montreal Cognitive Assessment (MoCA), Auditory Verbal Learning Test (AVLT), Shape Trail Test-A\&B (STT-A\&B) and Boston Naming Test.

1. General cognitive assessment: The MMSE and the basic MoCA will be used.

2. Memory assessment: The AVLT will be used. For the AVTL, there are 12 words in total that are repeated three times each. The short-delayed memory will be tested after 3-5 min and the long-delayed memory, clue memory and recognition after $20 \mathrm{~min}$. 
3. Perform function evaluation: The STT-A \& B will be used. Part A requires the subjects to connect randomly arranged numbers in order and at the fastest speed possible, while Part B requires the subjects to connect the numbers in order, and the two kinds of figures shall be carried out alternately. The longer it takes, the more severe the damage.

4. Language assessment: Fluency of animal words will be tested by measuring the number of animal names that can be provided in $1 \mathrm{~min}$. The Boston Naming Test will also be used. This test contains 30 items and the subjects must provide the names of the pictures that they see.

\section{MRI data acquisition}

MRI data will be acquired using a 3.0 Tesla Philips Achieva scanner at the HongQi Hospital, Mu Dan Jiang Medical University. For each participant, a conventional structural T1-weighted image (T1WI) will be obtained. Brain MRI will be inspected by an experienced neuroradiologist to flag individuals with structural brain abnormalities that need to be removed from further participation. T1WIs will be acquired with the following imaging parameters: $\mathrm{TR} / \mathrm{TE} / \mathrm{TI}=7 / 3.2 / 1100 \mathrm{~ms} ;$ flip angle $=7^{\circ} ; \mathrm{FOV}=256 \times$ $256 \mathrm{~mm}^{2}$; matrix size $=256 \times 256$; thickness $=1.0 \mathrm{~mm}$; $\mathrm{GAP}=0,192$ slices.

\section{MRI data analysis}

MRI data preprocessing will be performed using the SPM12 software package (http://www.fil.ion.Ucl.ac.uk/ $\mathrm{spm} /$ ). After routine preprocessing, voxel-based morphological analysis will be used to calculate the grey matter volume of each voxel in the whole brain. The Freesurfer image analysis software package (http://freesurfer.net/) will then be used to segment the hippocampal subregions and calculate their volumes.

\section{Analysis of NDEs}

At 06:00-08:00 in the morning on the day of examination, $10 \mathrm{~mL}$ of venous blood will be collected with an anticoagulant tube (including EDTA) and stored for $30 \mathrm{~min}$ at room temperature. After centrifugation at $2500 \mathrm{rpm}$ for 10 min at $4^{\circ} \mathrm{C}$, the supernatant will be taken as plasma and frozen at $-80^{\circ} \mathrm{C}$ for later analysis.

Exosomes will be extracted by ultrafast centrifugation. First, all cell fragments will be removed by centrifugation at $300 \mathrm{rpm}$ for $10 \mathrm{~min}$. Apoptotic cells will be removed after centrifugation at $2000 \mathrm{rpm}$ for $10 \mathrm{~min}$ and the remaining cell fragments and impurities will further be removed by centrifugation at $10000 \mathrm{rpm}$ for $20 \mathrm{~min}$. The supernatant will be centrifuged at $100000 \mathrm{rpm}$ for $70 \mathrm{~min}$ and then discarded. The precipitation will then be added to a phosphate buffered solution for suspension. The mouse anti-human CD171 antibody will be added to the exosome supernatant for incubation at $20^{\circ} \mathrm{C}$, and centrifuged at $400 \mathrm{rpm}$ for $5 \mathrm{~min}$. The supernatant will then be removed and the sediment resuspended in glycine $\mathrm{HCl}$ solution $(\mathrm{pH}=3.0)$, incubated for $10 \mathrm{~min}$ and centrifuged for $10 \mathrm{~min}$ at $4000 \mathrm{rpm}$. Tris- $\mathrm{HCl}(\mathrm{pH}=8.0)$, bovine serum albumin and mammalian protein extraction reagent will be added to the supernatant and stored separately at $-80^{\circ} \mathrm{C}$. An ELISA will finally be used to detect the content of Neurogranin, which will be standardised based on the number of exosomes.

Follow-ups with the participants will include the collection of T1WI MRI data, re-examination of the related neuropsychological scales, re-evaluation of the clinical diagnosis, analysis and processing of the data, extraction of the NEDs in the plasma and analysis of the neurogranin content.

\section{Statistical analysis}

Baseline neurogranin comparison and its correlation with cognition An analysis of variance will be used to compare the content of neurogranin NDEs in the three groups at baseline. General linear regression will be used to analyse the correlation between neurogranin content and baseline cognitive level by defining the cognitive score as the dependent variable and neurogranin content as the independent variable. Age, sex and years of education will be added to the model.

\section{Diagnostic accuracy of neurogranin}

A receiver operating curve analysis can easily determine the recognition ability of disease at any threshold value. This analysis will be used to clarify the changes of neurogranin in different stages of $\mathrm{AD}$ and to explore the diagnostic accuracy of neurogranin.

\section{Correlation between neurogranin and brain structure}

General linear regression will be used to analyse the effects of neurogranin on brain structure in the three groups by defining the whole brain volume and hippocampal subarea volume as dependent variables and neurogranin content as the independent variable. Age, sex and years of education will be added to the model.

\section{Correlation between neurogranin and cognitive decline}

General linear regression will be used to analyse the correlation between the level of neurogranin and the annual decline rate of cognitive function in the baseline period. Patients will be divided into four groups (quartiles) based on their neurogranin levels to determine the effect of neurogranin level on cognitive decline. To further explore whether there are differences in predicting cognitive decline between the healthy control group and the disease groups, the above-mentioned statistical analysis will be repeated across the three experimental groups. A similar analysis will be performed to determine the predictive effect of neurogranin on cognitive decline and whether it is concentration dependent.

\section{DISCUSSION}

This project will determine the role of neurogranin in the early diagnosis of $\mathrm{AD}$ by comparing the level of neurogranin plasma NEDs in three participant groups at baseline 
and by analysing the correlation thereof with cognition. In this project, we will analyse the correlation between neurogranin and brain structure-dependent content of NEDs, and explore its influence on brain atrophy, especially regarding hippocampal atrophy in patients with $\mathrm{AD}$.

It has been reported in a study of a normal elderly individuals that neurogranin is associated with age, $\mathrm{p}$-tau, t-tau and hippocampal volume, but not with amyloid protein. Furthermore, lower neurogranin content is associated with increased performance in delayed memory, and the correlation between delayed memory and neurogranin is independent of other biomarkers of $\mathrm{AD}{ }^{3}$ Moreover, the apolipoprotein E (APOE) gene is associated with neurogranin as it has been reported that APOEe4 carriers have a higher level of neurogranin than non-carriers in patient with aMCI. ${ }^{16}$ Although the correlation between neurogranin and tau protein is significant, the correlation between neurogranin and amyloid protein is weak ${ }^{6}$ as it is thought that neurogranin has a high binding specificity. This is often reflected in the varying levels of neurogranin across different neurological diseases.

Compared with Parkinson's disease and Lewy body dementia, the content of neurogranin in the CSF is higher in patients with $\mathrm{AD} .{ }^{17}$ Similarly, CSF neurogranin content has also been shown to be significantly higher in patients with $\mathrm{AD}$ than in patients with severe depression and in patients with non-AD-related MCI. ${ }^{18} 19$ A comparative study among various neurodegenerative diseases (frontotemporal dementia, Lewy body dementia, Parkinson's dementia, progressive supranuclear palsy and multiple system atrophy) found that the content of CSF neurogranin in patients with $\mathrm{AD}$ was significantly higher than in all other dysfunctions. Interestingly, when examining the dementia phenotype associated with these dysfunctions, there were no significant differences in such a behavioural variant. ${ }^{19}$ The area under the curve for distinguishing dementia associated with $\mathrm{AD}$ from that associated with other neurodegenerative dysfunctions was strong, at $0.76 .^{20}$

Although there have been few studies that found no significant changes in plasma neurogranin between patients with $\mathrm{AD}$ and healthy controls, ${ }^{21}$ with the development of plasma NDE detection technology, we can now detect changes of neurodegeneration-related neuronal proteins in patients with $\mathrm{AD} .{ }^{10}{ }^{11}$ It has been found that NDEs in plasma decrease in patients with $\mathrm{AD}$ and are also suppressed several years before disease onset. ${ }^{14}$ Furthermore, other studies have found that an elevated level of neurogranin is associated with a smaller hippocampal volume. ${ }^{3}$ As far as we know, pathological findings of $\mathrm{AD}$ show that senile plaques and neurofibrillary tangles first appear in the olfactory cortex and hippocampus. The hippocampus is an important part of the cholinergic circuit of the septal area and is closely related to learning and memory in mammals. Lesion studies involving the hippocampus have provided the anatomical basis for memory impairment in patients with $\mathrm{AD}^{22}$ In our primary study, we found that such a trend changes in the hippocampus subareas and can provide further detail to illustrate the preclinical stages of AD. Future studies are needed to incorporate longitudinal designs and multiple biomarkers to fully assess these stages.

Here, the present study aims to develop a new blood marker for $\mathrm{AD}$ and to determine the correlation between plasma NDEs and the volume of the hippocampus during the progression of aMCI to AD. Additionally, we hope to provide scientific evidence for a new prevention, diagnosis and treatment protocol of AD.

\section{Ethics approval and consent to participate}

Ethical approval was obtained from the medical research ethics committee and institutional review board of HongQi Hospital, Mudanjiang Medical University. All participation is based on written informed consent and the participants will be able to withdraw from the study at any time.

Acknowledgements The authors would like to thank the participants of this study and patient advisers for their support.

Contributors $\mathrm{MH}$ and LS are joint first authors. CY and WC are responsible for the design. MH and LT are responsible for the manuscript draft and revision. WS and $\mathrm{PL}$ are responsible for the implementation of the trial. ZX and $\mathrm{MH}$ are responsible for the implementation of imaging data analysis. WZ is responsible for the study design, implementation of the trial, manuscript draft and revision, and funding. All authors contributed to the drafting of the manuscript and approved the final manuscript.

Funding This study was supported by the Foundation of HongQi (2018HQ-01), the National Natural Science Foundation of China (81771795), the Mudanjiang Science and Technology Project (Z2018s051, Z2018s065), the Major Project of Postgraduate Innovation Research Projects in Heilongjiang Province(2017YJSCX05MY),the Postgraduate Innovation Fund Project of Mudanjiang Medical University (2018YJSCX-21MY) and the Scientific Research Projects of Heilongjiang Provincial Universities (2019-KYYWFMY-0029).

Competing interests None declared.

Patient consent for publication Not required.

Provenance and peer review Not commissioned; externally peer reviewed.

Open access This is an open access article distributed in accordance with the Creative Commons Attribution Non Commercial (CC BY-NC 4.0) license, which permits others to distribute, remix, adapt, build upon this work non-commercially, and license their derivative works on different terms, provided the original work is properly cited, appropriate credit is given, any changes made indicated, and the use is non-commercial. See: http://creativecommons.org/licenses/by-nc/4.0/.

ORCID iD

MengFei He http://orcid.org/0000-0002-8191-5231

\section{REFERENCES}

1 Keogh-Brown MR, Jensen HT, Arrighi HM, et al. The impact of Alzheimer's disease on the Chinese economy. EBioMedicine 2016;4:184-90.

2 Forner S, Baglietto-Vargas D, Martini AC, et al. Synaptic impairment in Alzheimer's disease: a dysregulated symphony. Trends Neurosci 2017;40:347-57.

3 Casaletto KB, Elahi FM, Bettcher BM, et al. Neurogranin, a synaptic protein, is associated with memory independent of Alzheimer biomarkers. Neurology 2017;89:1782-8.

4 Tarawneh R, D'Angelo G, Crimmins D, et al. Diagnostic and prognostic utility of the synaptic marker neurogranin in Alzheimer disease. JAMA Neurol 2016;73:561.

5 Wellington $\mathrm{H}$, Paterson RW, Suárez-González A, et al. CSF neurogranin or tau distinguish typical and atypical Alzheimer disease. Ann Clin Transl Neurol 2018;5:162-71. 
6 Lista S, Toschi N, Baldacci F, et al. Cerebrospinal fluid neurogranin as a biomarker of neurodegenerative diseases: a cross-sectional study. $J$ Alzheimers Dis 2017;59:1327-34.

7 Portelius E, Zetterberg H, Skillbäck T, et al. Cerebrospinal fluid neurogranin: relation to cognition and neurodegeneration in Alzheimer's disease. Brain 2015;138:3373-85.

8 Kester MI, Teunissen CE, Crimmins DL, et al. Neurogranin as a cerebrospinal fluid biomarker for synaptic loss in symptomatic Alzheimer disease. JAMA Neurol 2015;72:1275-80.

9 Headley A, De Leon-Benedetti A, Dong C, et al. Neurogranin as a predictor of memory and executive function decline in $\mathrm{MCl}$ patients. Neurology 2018;90:e887-95.

10 De Vos A, Jacobs D, Struyfs H, et al. C-terminal neurogranin is increased in cerebrospinal fluid but unchanged in plasma in Alzheimer's disease. Alzheimers Dement 2015;11:1461-9.

11 Goetzl EJ, Boxer A, Schwartz JB, et al. Altered lysosomal proteins in neural-derived plasma exosomes in preclinical Alzheimer disease. Neurology 2015;85:40-7.

12 Fiandaca MS, Kapogiannis D, Mapstone M, et al. Identification of preclinical Alzheimer's disease by a profile of pathogenic proteins in neurally derived blood exosomes: a case-control study. Alzheimers Dement 2015;11:600-7. e601.

13 Abner EL, Jicha GA, Shaw LM, et al. Plasma neuronal exosomal levels of Alzheimer's disease biomarkers in normal aging. Ann Clin Transl Neurol 2016;3:399-403.

14 Goetzl EJ, Kapogiannis D, Schwartz JB, et al. Decreased synaptic proteins in neuronal exosomes of frontotemporal dementia and Alzheimer's disease. Faseb J 2016;30:4141-8.
15 den Heijer T, van der Lijn F, Koudstaal PJ, et al. A 10-year follow-up of hippocampal volume on magnetic resonance imaging in early dementia and cognitive decline. Brain 2010;133:1163-72.

16 Sun X, Dong C, Levin B, et al. APOE $\varepsilon 4$ carriers may undergo synaptic damage conferring risk of Alzheimer's disease. Alzheimers Dement 2016;12:1159-66.

17 Remnestål J, Just D, Mitsios N, et al. CSF profiling of the human brain enriched proteome reveals associations of neuromodulin and neurogranin to Alzheimer's disease. Proteomics Clin Appl 2016;10:1242-53.

18 Hellwig K, Kvartsberg $\mathrm{H}$, Portelius E, et al. Neurogranin and YKL-40: independent markers of synaptic degeneration and neuroinflammation in Alzheimer's disease. Alzheimers Res Ther 2015;7:1-8.

19 Sanfilippo C, Forlenza O, Zetterberg $\mathrm{H}$, et al. Increased neurogranin concentrations in cerebrospinal fluid of Alzheimer's disease and in mild cognitive impairment due to AD. J Neural Transm 2016;123:1443-7.

20 Janelidze S, Hertze J, Zetterberg H, et al. Cerebrospinal fluid neurogranin and YKL-40 as biomarkers of Alzheimer's disease. Ann Clin Transl Neurol 2016;3:12-20.

21 Kvartsberg H, Portelius E, Andreasson U, et al. Characterization of the postsynaptic protein neurogranin in paired cerebrospinal fluid and plasma samples from Alzheimer's disease patients and healthy controls. Alzheimers Res Ther 2015;7:1-9.

22 Thompson PM, Mega MS, Woods RP, et al. Cortical change in Alzheimer's disease detected with a disease-specific populationbased brain atlas. Cereb Cortex 2001;11:1-16. 